\title{
Global Existence to an Attraction-Repulsion Chemotaxis Model with Fast Diffusion and Nonlinear Source
}

\author{
Yingjie Zhu ${ }^{1,2}$ and Fuzhong Cong ${ }^{1,3}$ \\ ${ }^{1}$ Institute of Mathematics, Jilin University, Changchun 130012, China \\ ${ }^{2}$ Institute of Science, Changchun University, Changchun 130022, China \\ ${ }^{3}$ Aviation University of Air Force, Changchun 130022, China \\ Correspondence should be addressed to Fuzhong Cong; congfz67@126.com
}

Received 7 April 2015; Revised 20 June 2015; Accepted 12 July 2015

Academic Editor: Michael Radin

Copyright (C) 2015 Y. Zhu and F. Cong. This is an open access article distributed under the Creative Commons Attribution License, which permits unrestricted use, distribution, and reproduction in any medium, provided the original work is properly cited.

\begin{abstract}
This paper deals with the global existence of solutions to a strongly coupled parabolic-parabolic system of chemotaxis arising from the theory of reinforced random walks. More specifically, we investigate the attraction-repulsion chemotaxis model with fast diffusive term and nonlinear source subject to the Neumann boundary conditions. Such fast diffusion guarantees the global existence of solutions for any given initial value in a bounded domain. Our main results are based on the method of energy estimates, where the key estimates are obtained by a technique originating from Moser's iterations. Moreover, we notice that the cell density goes to the maximum value when the diffusion coefficient of the cell density tends to infinity.
\end{abstract}

\section{Introduction}

Chemotaxis is known as the active orientation of moving organisms along the chemical gradient. It is observed in many natural systems. For example, myxobacteria produce so-called slime trails on which their cohorts can move more readily. The mathematical models of chemotaxis were introduced by Patlak in [1] and Keller and Segel in [2]. During the past four decades, chemotaxis models have been studied extensively (see, e.g., [3-10] and the rich references therein). For instance, Othmer and Stevens in [3] modeled myxobacteria as individual random walkers and proposed the microscopic model based on the velocity jump process. By taking the parabolic limit of microscopic model, we can obtain the macroscopic chemotaxis model which is the well-known Keller-Segel system:

$$
\begin{aligned}
u_{t}=\nabla \cdot(\mu(u) \nabla u)-\nabla \cdot(\chi u \nabla \Theta(v))+f(u), & \\
x \in \Omega, t>0, & \\
\tau v_{t}=d \Delta v+\epsilon u-\beta v, \quad x \in \Omega, t>0, &
\end{aligned}
$$

where $\Omega \subset \mathbb{R}^{n}$ is a bounded connected domain with a smooth boundary $\partial \Omega, \epsilon>0, \beta>0$, and $\chi>0$ are positive constants, and $\tau=0,1$. The function $u=u(x, t)$ denotes the cell density and $v=v(x, t)$ represents the chemotactic concentration, for example, the oxygen. The constant $\chi$ is called the chemosensitive coefficient, and the sign of $\chi$ corresponds to chemoattraction if $\chi>0$, and chemorepulsion if $\chi<0$. The function $\mu(u)$ and the constant $d$ are the diffusion coefficient of the cell motility and the chemical, respectively. The function $f(u)$ represents the kinetic function describing production and degradation of chemicals, and $\Theta(v)$ is commonly referred to the chemotactic potential function.

In the absence of logistic source (i.e., $f(u) \equiv 0$ ), there have been extensive studies to system (1). The main feature of solution to the Keller-Segel model is the possibility of blowup in finite time in $[3,8,11,12]$. For instance, the first result on finite-time blowup for a radially symmetric solutions was shown in $[13,14]$ when $\Omega$ is a ball in $\mathbb{R}^{2}$ under the condition that $\int_{\Omega} u_{0} d x>8 \pi /(\epsilon \chi)$ and $\int_{\Omega} u_{0}(x)|x|^{2} d x$ is sufficiently small. For a general domain, Nagai in [15] further showed the finite-time blowup of nonradial solutions provided that $\int u_{0}|x-y|^{2} d x$ is sufficiently small and $\int_{\Omega} u_{0} d x>8 \pi /(\epsilon \chi)$ if $y \in \Omega$ or $\int_{\Omega} u_{0} d x>4 \pi /(\epsilon \chi)$ if $y \in \partial \Omega$ with $\tau=$ 1. Winkler in [16] studied finite-time blowup of radially symmetric solutions to the full parabolic system with logistic 
sources $f(u)=\lambda u-\zeta u^{\kappa}$ in a ball $\Omega \subset \mathbb{R}^{n}$ with parameters $\lambda \geq$ $0, \zeta \geq 0$, and $\kappa \geq 1$. Moreover, Winkler in [8] gave the set of blowup to the system (1) by enforcing initial data with respect to the topology of $L^{p}(\Omega) \times W^{1,2}(\Omega)$ for any $p \in(1,2 n /(n+2))$, where $\Omega$ is a ball in $\mathbb{R}^{n}$ with $n \geq 3$. In summary, the solution of system (1) never blows up when $n=1$, whereas there is finite-time or infinite-time blowup when $n \geq 3$. Moreover, recent results in [17] confirmed that the attraction-repulsion is a plausible mechanism to regularize the classical KellerSegel system (2) with $f(u)=0$ whose solutions may blow up in higher dimensions. Authors in $[5,9,10,18,19]$ proved the global existence of solutions.

However, in many biology progresses, the cells usually interact with not only the attractive combination but also repulsive signalling. Therefore, it is necessary to study the attraction-repulsion chemotaxis model:

$$
\begin{aligned}
u_{t}=\Delta u-\nabla \cdot(\chi u \nabla v)+\nabla \cdot(\xi u \nabla w)+f(u), & \\
x \in \Omega, t>0, & \\
\tau v_{t}=\Delta v+\epsilon u-\beta v, \quad x \in \Omega, t>0, & \\
\tau w_{t}=\Delta w+\kappa u-\delta v, \quad x \in \Omega, t>0 . &
\end{aligned}
$$

System (2) with $f(u)=0$ was proposed in [20] to describe the aggregation of microglia observed in Alzheimer's disease. Here $u(x, t)$ represents the cell density and $v(x, t)$ denotes the concentration of the chemoattractant and $w(x, t)$ is the concentration of the chemorepulsion. The constants satisfy $\chi \geq 0$ and $\xi \geq 0$, where $\chi$ and $\xi$ measure the strength of chemotactic signal of attraction and repulsion, respectively. The constants $\epsilon, \beta, \kappa$, and $\delta$ are positive, and they denote the production and degradation rates of the two chemicals, respectively. The first cross-diffusive term in the first equation of (2) means that the orientation movement of the cell is directed to the chemorepulsion, whereas the second crossdiffusive term implies that cells move down the chemoattraction. The second and third equations in (2) elaborate that the two chemicals of chemoattraction and chemorepulsion are released by cells and go through decay. For the case of $f(u) \equiv$ 0 , the theorem of competing effects has been established in [17] with $\tau=0$. Moreover, the global existence, asymptotic behavior, and steady states of classical solution were studied in [21] for one-dimensional case with $\tau=1$.

\section{Motivation}

In this paper, we consider the following attraction-repulsion chemotaxis system including three parabolic equations:

$$
\begin{gathered}
u_{t}=\nabla \cdot(\mu(u) \nabla u)-\nabla \\
\cdot\left(\chi_{v}(u, v) u \nabla v+\chi_{w}(u, w) u \nabla w\right)+f(u), \\
x \in \Omega, t>0, \gamma \geq 2, \\
v_{t}=d_{v} \Delta v+g(u, v), \quad x \in \Omega, t>0, \\
w_{t}=d_{w} \Delta w+h(u, w), \quad x \in \Omega, t>0,
\end{gathered}
$$

with the initial-boundary value conditions:

$$
\begin{aligned}
& u(x, 0)=u_{0}, \quad x \in \Omega, \\
& v(x, 0)=v_{0}, \quad x \in \Omega, \\
& w(x, 0)=w_{0}, \quad x \in \Omega, \\
& \left.\frac{\partial u}{\partial \nu}\right|_{\partial \Omega}=\left.\frac{\partial v}{\partial \nu}\right|_{\partial \Omega}=\left.\frac{\partial w}{\partial \nu}\right|_{\partial \Omega}=0, \quad x \in \partial \Omega, t>0,
\end{aligned}
$$

where $\Omega \subset \mathbb{R}^{n}$ is a bounded $C^{2+\eta}$ domain for some $0<$ $\eta<1$ and $v$ denotes the unit outer normal vector field on $\partial \Omega$. The function of the cell density $u=u(x, t)$ is the fraction of volume occupied by cells, whereas the fast diffusion coefficient of the cell is described as

$$
\mu(u)=\frac{1}{(1-u)^{\gamma}}, \quad \gamma \geq 2 .
$$

It is easy to see that the function $1 /(1-u)^{\gamma}$ is a monotonically increasing function of $u$ which guarantees that the solution will not blow up. The functions $v=v(x, t)$ and $w=$ $w(x, t)$ denote the concentration of the chemoattraction and chemorepulsion, respectively. The constants $d_{v}>0$ and $d_{w}>0$ are the diffusion coefficients of the chemoattraction and chemorepulsion, respectively. $\chi_{v}$ and $\chi_{w}$ denote the chemosensitive coefficients, and $f(u)$ describes the kinetic function.

In particular, the global existence of solutions was obtained by increasing the diffusion coefficient $\mu(u)$ : for example, $\mu(u)=1 /(1-u)^{\gamma}, \gamma \geq 2$ in [11], and $\mu(u)=$ $(1+u) /(1-u+\log u)$ in [5]. Liu and Tao in [22] established global boundedness of classical solutions to the parabolic attraction-repulsion chemotaxis system (2) when $f(u) \equiv 0$. For the case of including logistic source, global existence and boundedness of classical solution were studied in $[23,24]$. For the case $\gamma=1$, the existence of families of traveling impulses and fronts was analyzed in [25].

In this paper, we will prove the existence of global classical solutions to the generalized system (3) with the initialboundary conditions and the no-flux boundary condition (4). In addition, we need the following assumptions.

(A1) Let $\mathscr{D}:=[0,1] \times[0,+\infty)$. Suppose that functions $\chi_{v}(u, v), \chi_{w}(u, w): \mathscr{D} \rightarrow[0,+\infty)$ are of $C^{2}$ class, and $\chi_{v}(0, v)=0$ for $v \geq 0$ and $\chi_{w}(0, w)=0$ for $w \geq 0$.

(A2) Assume that functions $f, g, h: \mathscr{D} \rightarrow R$ are of $C^{2}$ class and that $f(u) \leq M_{f}(1-u), g(u, v) \leq M_{g}(1-u)$, and $h(u, w) \leq M_{h}(1-u)$ for all $(u, v),(u, w) \in \mathscr{D}$, where $M_{f}, M_{g}, M_{h} \geq 0$.

(A3) Let $d_{v}, d_{w}:[0,+\infty) \rightarrow\left[d_{0}, d_{1}\right)$ be of $C^{2}$ class, where $0<d_{0}<d_{1}<\infty$

(A4) $u_{0}, v_{0}, w_{0} \in C(\bar{\Omega}), 0 \leq u_{0}(x)<1$, and $v_{0}(x), w_{0}(x) \geq$ 0 for $x \in C(\bar{\Omega})$ and $\partial u_{0} / \partial v=\partial v_{0} / \partial v=\partial w_{0} / \partial v=0$ for $x \in \partial \Omega$.

The main result is the following. 
Theorem 1. Let $\left(u_{0}, v_{0}, w_{0}\right) \in\left[H^{2}(\Omega)\right]^{3}$. Then there exists a unique global triple solution of $(u, v, w)$ to system (3) satisfying initial conditions (4) and

$$
\begin{aligned}
(u, v, w) \in[ & {\left[C^{0}\left(\bar{\Omega} \times[0,+\infty) ; \mathbb{R}^{3}\right)\right]^{3} } \\
& \cap\left[C^{2,1}\left(\bar{\Omega} \times(0,+\infty) ; \mathbb{R}^{3}\right)\right]^{3} .
\end{aligned}
$$

Furthermore, there exist two constants $C_{v} \geq 0$ and $C_{w} \geq 0$ such that

$$
\begin{aligned}
& 0 \leq v(x, t) \leq C_{v}, \\
& 0 \leq w(x, t) \leq C_{w}, \\
& 0 \leq u(x, t)<1 .
\end{aligned}
$$

Throughout this paper, we introduce some notations. $\Omega$ is a bounded open interval in $\mathbb{R}=(-\infty,+\infty)$ and $C$ denotes a general constant which may have different values in different place. $L^{p}=L^{p}(\Omega)(1 \leq p<\infty)$ is the usual Lebesgue space with the norm $\|f\|_{L^{p}}=\left(\int_{\Omega}|f(x)|^{p}\right)^{1 / p}$ for $1 \leq p<\infty$ and $\|f\|_{L^{\infty}}=\operatorname{esssup}_{x \in \Omega}|f|$. When $p=2$, we denote $\|f\|_{L^{2}}=$ $\|f\|$ for convenience. $H^{k}$ is the $k$ th-order Sobolev space $W^{k, 2}$ with the norm $\|f\|_{H^{k}}=\left(\sum_{\alpha=0}^{k}\left\|\partial_{x}^{\alpha} f(x)\right\|^{2}\right)^{1 / 2}$. For notational convenience, we write $\|f(\cdot, t)\|_{L^{p}}$ and $\|f(\cdot, t)\|_{H^{k}}$ as $\|f(\cdot, t)\|_{p}$ and $\|f(\cdot, t)\|_{k}$, respectively. Moreover, we denote

$$
\begin{gathered}
\|(f, g, h)\|_{p}=\|f\|_{p}+\|g\|_{p}+\|h\|_{p}, \quad 1 \leq p \leq+\infty, \\
\|(f, g, h)\|_{k}=\|f\|_{k}+\|g\|_{k}+\|h\|_{k}, \quad k=1,2,3, \ldots
\end{gathered}
$$

The rest of this paper is organized as follows. In Section 2, we establish the local existence and give some preliminary lemmas. Some necessary priori estimates will be established in Section 3. We will complete the proof of Theorem 1 in Section 4.

\section{Local Existence}

To prove Theorem 1, we need to establish local existence of solutions to system (3) and some priori estimates in this section.

Theorem 2 (local existence). Let assumptions (A1)-(A4) hold. Then

(1) There is a positive constant $T_{0}$ depending on initial value $\left(u_{0}, v_{0}, w_{0}\right)$ such that system (3) has a unique maximal solution $(u, v, w)$ in the space $C^{1+q / 2,2+q}(\bar{\Omega} \times$ $\left.\left[0, T_{0}\right) ; \mathbb{R}^{3}\right)$ with $u(x, t), v(x, t), w(x, t) \geq 0$.

(2) There is a global classical solution of system (3) if $u(x, t)$ is bounded away from 1 for each finite time $t>0$, which means $T_{0}=+\infty$.

Proof. Let $U=(u, v, w) \in \mathbb{R}^{3}$. System (3) can be written as

$$
\partial_{t} \mathbf{U}=\nabla \cdot(J(\mathbf{U}) \nabla \mathbf{U})+\mathscr{F}(\mathbf{U})
$$

where

$$
\begin{aligned}
J(\mathbf{U}) & =\left[\begin{array}{ccc}
d_{u} & -\chi_{v} u & \chi_{w} u \\
0 & d_{v} & 0 \\
0 & 0 & d_{w}
\end{array}\right], \\
\mathscr{F}(\mathbf{U}) & =\left[\begin{array}{c}
f(u) \\
g(u, v) \\
h(u, w)
\end{array}\right] .
\end{aligned}
$$

System (9) satisfies the initial value

$$
\mathbf{U}(\cdot, 0)=\left(u_{0}, v_{0}, w_{0}\right), \quad x \in \Omega .
$$

For some $\theta>0$ and given initial conditions $u_{0}<1-\theta$, it is clear that the eigenvalues of matrix $J(\mathbf{U})$ are positive at $t=0$. Thus, system (9) is normally parabolic and there exists local solution by Theorem 7.3 in [26]; that is, there is a $T_{0}>0$ such that the unique solution $(u, v, w) \in C\left(\left[0, T_{0}\right) \times \bar{\Omega} ; \mathbb{R}^{3}\right) \cap$ $C^{1,2}\left(\left[0, T_{0}\right) \times \bar{\Omega} ; \mathbb{R}^{3}\right)$.

Next we rewrite the first equation of (3) as

$$
\begin{aligned}
u_{t} & =\frac{1}{(1-u)^{\gamma}} \Delta u+\left(\frac{\gamma}{(1-u)^{\gamma+1}} \nabla u-\chi_{v u} \nabla v\right. \\
& \left.+\chi_{w u} \nabla w\right) \cdot \nabla u-\left(\chi_{v} \Delta v+\chi_{w} \Delta w+\chi_{v v}|\nabla v|^{2}\right. \\
& \left.+\chi_{w w}|\nabla w|^{2}+f(u)\right) .
\end{aligned}
$$

Recalling that assumption (A2) ensures that $\chi_{v}(0, v)=0$ for all $v \geq 0$ and $\chi_{w}(0, w)=0$ for all $w \geq 0$, this means that $\chi_{v v}(0, v)=\chi_{w w}(0, w)=0$ for all $v \geq 0$ and $w \geq 0$, where $\chi_{v v}=\partial \chi_{v} / \partial v$ and $\chi_{w w}=\partial \chi_{w} / \partial w$. Hence, the source term $\chi_{v} \Delta v+\chi_{w} \Delta w+\chi_{v v}|\nabla v|^{2}+\chi_{w w}|\nabla w|^{2}+f(u)=0$ is zero when $u=0$ at some $\left(x_{0}, t_{0}\right)$. By applying the maximum principle to $(1.1)_{a}$, therefore, we obtain that $u \geq 0$ whenever $v \geq 0$ and $w \geq 0$. Similarly, we infer $v \geq 0$ (or $w \geq 0$ ) from the second equation of (3) (or the third of (3)) whenever $u \geq 0$. The proof of (1) in Theorem 2 is finished.

The proof of (2) in Theorem 2 is completed by applying the theorem of quasilinear parabolic equations in [26] since system (9) is an upper triangular system.

\section{A Priori Estimates}

Next, we recall the Gagliardo-Nirenberg inequality for functions satisfying the boundary condition for $t>0$ (see [27, 28]).

Lemma 3. Let $\Omega$ be a bounded open domain satisfying the uniform cone property in $\mathbb{R}^{n}$ with $n \geq 2$ :

$$
p \in \begin{cases}{[1,+\infty),} & \text { if } n=2, \\ {\left[1, \frac{2 n}{n-2}\right),} & \text { if } n \geq 3 .\end{cases}
$$

Then there exists a positive constant $C_{p}$, which depends on $n$, $p$, and $\Omega$, such that for all $u \in W^{1,2}$ :

$$
\|u\|_{p} \leq C_{p}\left(\|\nabla u\|_{2}^{a}\|u\|_{1}^{1-a}+\|u\|_{1}\right),
$$

where $a=(1-1 / p) /(1 / 2+1 / n)$ and $0 \leq a<1$. 
Lemma 4. Let the conditions in Lemma 3 hold: $a_{0}=n /(n+2)$ and $l \geq \gamma$. Then there exists a constant $N_{1}$ independent of $l$ such that

$$
\begin{aligned}
\|u\|_{2 l /(l+\gamma)} \leq N_{1}\left(\|\nabla u\|_{2}^{a_{0}(l-\gamma / l)}\|u\|_{1}^{1-a_{0}(l-\gamma) / l}+\|u\|_{1}\right) & \\
\forall u & \in W^{1,2}(\Omega) .
\end{aligned}
$$

Proof. Recalling $1 \leq 2 l /(l+\gamma)<2$ for $l \geq \gamma$ and employing Lemma 3 with $a=(1-1 / p) /(1 / 2+1 / n)$ and $p=2$, we obtain $a_{0}=n /(n+2)$; thus,

$$
\begin{aligned}
\|u\|_{2 l /(l+\gamma)} & \leq\left[C_{q}\|\nabla u\|_{2}^{a_{0}}\|u\|_{1}^{1-a_{0}}+\|u\|_{1}\right]^{(l-\gamma) / l}\|u\|_{1}^{\gamma / l} \\
& \leq N_{1}\left(\|\nabla u\|_{2}^{a_{0}(l-\gamma) / l}\|u\|_{1}^{1-a_{0}(l-\gamma) / l}+\|u\|_{1}\right),
\end{aligned}
$$

where we have used the inequality $(c+d)^{m} \leq 2^{m-1}\left(c^{m}+d^{m}\right)$ for $c, d, m \geq 0$.

Lemma 5. Let a nonnegative numerical sequence $\left\{S_{i}\right\}_{i=0}^{\infty}$ satisfying $S_{i+1} \leq a_{i} S_{i}^{1+b_{i}}$ with $a_{i} \geq 1$ and $b_{i} \geq 0$ for $i \geq 0$. Suppose $S_{0} \geq 1$ and $\prod_{i=1}^{\infty}\left(1+b_{i}\right)=N_{2}<+\infty$. Then one has

$$
S_{i} \leq\left(\prod_{i=0}^{\infty} a_{i}\right)^{N_{2}} S_{0}^{N_{2}} .
$$

Proof. By the definition of the sequence $\left\{S_{i}\right\}_{i=0}^{\infty}$, we obtain that for $\mathrm{N}_{2} \geq 1$

$$
\begin{aligned}
S_{i} & \leq a_{i-1} S_{i-1}^{1+b_{i-1}} \leq a_{i-1}\left(a_{i-2} S_{i-2}^{1+b_{i-2}}\right)^{1+b_{i-1}} \\
& =a_{i-1} a_{i-2}^{1+b_{i-1}} S_{i-2}^{\left(1+b_{i-2}\right)\left(1+b_{i-1}\right)} \\
& \leq a_{i-1} a_{i-2}^{1+b_{i-1}} a_{i-3}^{\left(1+b_{i-1}\right)\left(1+b_{i-2}\right)} S_{i-3}^{\left(1+b_{i-3}\right)\left(1+b_{i-2}\right)\left(1+b_{i-1}\right)} \\
& \leq a_{i-1} a_{i-2}^{N_{2}} a_{i-3}^{N_{2}} S_{i-3}^{\left(1+b_{i-3}\right)\left(1+b_{i-2}\right)\left(1+b_{i-1}\right)} \leq \cdots \\
& \leq\left(\prod_{i=0}^{\infty} a_{i}\right)^{N_{2}} S_{0}^{N_{2}} .
\end{aligned}
$$

Lemma 6. Let conditions (A1)-(A4) hold. Then there exists a unique global solution $(u, v, w)$ to system (3) such that $u, v$, and $w$ are in $C^{1+\eta / 2,2+\eta}\left(\bar{\Omega} \times[0,+\infty) ; \mathbb{R}^{3}\right)$. Furthermore, there exists two positive constants $C_{v}$ and $C_{w}$ satisfying

$$
\begin{aligned}
& 0 \leq v(x, t) \leq C_{v}, \\
& 0 \leq w(x, t) \leq C_{w}, \\
& 0 \leq u(x, t)<1
\end{aligned}
$$

$$
\forall x \in \bar{\Omega}, \forall t \geq 0 .
$$

Proof. After appropriate scaling, the system is limited in a region $\Omega$ satisfying $|\Omega|=1$. Next, we introduce the auxiliary scalar equation:

$$
\begin{aligned}
\partial_{t} u= & \nabla \cdot\left(\frac{1}{(1-u)^{\alpha}} \nabla u\right)-\nabla \cdot \vec{b}(x, t)+\nabla \cdot \vec{c}(x, t) \\
& +\vec{P}(u, x, t) \quad \forall x \in \Omega \times[0, T)
\end{aligned}
$$

with the initial-boundary condition:

$$
\begin{aligned}
u(x, 0) & =u_{0}(x), \quad \forall x \in \Omega \\
\frac{\partial u}{\partial \nu} & =0, \\
\left.\vec{b} \cdot \vec{v}\right|_{\partial \Omega} & =0, \\
\left.\vec{c} \cdot \vec{v}\right|_{\partial \Omega} & =0
\end{aligned}
$$

$\forall x \in \partial \Omega$,

where $\vec{b}(x, t), \vec{c}(x, t)$, and $\vec{P} \in L^{\infty}(\Omega \times(0, \infty))$ are given functions. To complete the proof of Lemma 6 , we will establish the following lemma.

Lemma 7. Suppose $0 \leq u_{0}(x) \leq 1$ for all $x \in \Omega$. Let

$$
\begin{gathered}
\|\vec{b}\|_{L^{\infty}(\Omega \times(0,+\infty))}=M_{b}, \\
\|\vec{c}\|_{L^{\infty}(\Omega \times(0,+\infty))}=M_{c}, \\
\|\vec{P}(u, \cdot, \cdot)\|_{L^{\infty}(\Omega \times(0,+\infty))} \leq M_{P}(1-u)
\end{gathered}
$$

for $u \in[0,1)$.

Let $u$ be a classical solution to system (20)-(21) satisfying $0 \leq$ $u_{0}(x)<1$ for $(x, t) \in Q_{T}=\bar{\Omega} \times[0, T)$. Then, for any $T>0$, there exists a constant $Q_{T}>0$ such that $u(x, t)<1-\varepsilon_{T}$ for all $(x, t) \in Q_{T}$, where the constant $\varepsilon_{T}$ depends only on $M_{b}, M_{c}$, $\theta=\left\|1-u_{0}\right\|_{L^{\infty}(\Omega)}$, and $T$.

Proof. We take $\theta>0$ satisfying $u_{0} \leq 1-\theta$ for all $x \in \bar{\Omega}$ and notice that $|\Omega|=1$ by scaling $x$ and $t$ in the governing equation. To complete the proof, we have to divide the proof into three steps.

Step 1 . We will firstly prove that $1 /(1-u) \in L^{q}(\Omega)$ for any $1 \leq q<\infty$. Multiplying both sides of the first equation of (3) by $q(1-u)^{-q-1}$ and integrating the resultant equation, we yield

$$
\frac{d}{d t} \int(1-u)^{-q} d x=\int q(1-u)^{-q-1} u_{t} d x=q \int(1
$$

$$
\begin{aligned}
& -u)^{-q-1} \nabla\left[\frac{\nabla u}{(1-u)^{\gamma}}-\vec{b}(x, t)+\vec{c}(x, t)\right] d x \\
& +q \int(1-u)^{-q-1} \vec{P}(u, x, t) d x=-q(1+q) \\
& \cdot \int\left(\frac{|\nabla u|^{2}}{(1-u)^{\gamma+P+2}}-\frac{\nabla u \cdot(\vec{b}(x, t)-\vec{c}(x, t))}{(1-u)^{q+2}}\right) d x
\end{aligned}
$$




$$
\begin{aligned}
& +q \int \frac{\vec{P}(u, x, t)}{(1-u)^{q+1}} d x \leq-q(1+q) \int\left(\frac{|\nabla u|^{2}}{(1-u)^{\gamma+P+2}}\right. \\
& \left.-\frac{\nabla u \cdot(\vec{b}(x, t)-\vec{c}(x, t))}{(1-u)^{q+2}}\right) d x \\
& +q \int \frac{M_{P}}{(1-u)^{q}} d x
\end{aligned}
$$

where the last inequality follows from (A3); then (23) is rewritten as

$$
\begin{aligned}
& \frac{d}{d t} \int(1-u)^{-q} d x-q \int \frac{M_{P}}{(1-u)^{q}} d x \leq q(1+q) \\
& \quad \cdot \int-\left|\frac{\nabla u}{(1-u)^{(\gamma+q+2) / 2}}\right|^{2} \\
& \left.+\frac{\nabla u \cdot(\vec{b}(x, t)-\vec{c}(x, t))}{(1-u)^{(\gamma+q+2) / 2}} \cdot \frac{1}{(1-u)^{(q+2-\gamma) / 2}}\right] d x .
\end{aligned}
$$

Noticing $W_{q}=(1-u)^{-(\gamma+q) / 2}$, we obtain the fact that

$$
\begin{aligned}
(1-u)^{-q} & =W_{q}^{2 q /(\gamma+q)}, \\
\nabla W_{q} & =\frac{\gamma+q}{2}(1-u)^{-(\gamma+q+2) / 2} \nabla u .
\end{aligned}
$$

Substituting (24) into (25), we have

$$
\begin{aligned}
& \frac{d}{d t} \int(1-u)^{-q} d x-q \int \frac{M_{P}}{(1-u)^{q}} d x \leq q(1+q) \\
& \cdot \int\left[-\frac{4}{(\gamma+q)^{2}}\left|\nabla W_{q}\right|^{2}+\frac{2}{\gamma+q}\right. \\
& \cdot \frac{\nabla W_{q} \cdot(\vec{b}(x, t)-\vec{c}(x, t))}{\left.(1-u)^{(q+2-\gamma) / 2}\right] d x=\frac{q(1+q)}{\gamma+q}} \\
& \cdot \int\left[-\frac{4}{\gamma+q}\left|\nabla W_{q}\right|^{2}\right. \\
& \left.+\frac{2 \nabla W_{q} \cdot(\vec{b}(x, t)-\vec{c}(x, t))}{(1-u)^{(q+2-\gamma) / 2}}\right] d x \leq \frac{q(1+q)}{\gamma+q} \\
& +\int-\frac{4}{\gamma+q}\left|\nabla W_{q}\right|^{2} d x \\
& +2 \int \frac{\nabla W_{q}\left(M_{b}-M_{c}\right)}{(1-u)^{(q+2-\gamma) / 2}} d x \leq \frac{q(1+q)}{\gamma+q}
\end{aligned}
$$

$$
\begin{aligned}
& \int-\frac{4}{\gamma+q}\left|\nabla W_{q}\right|^{2} d x+2 \int\left(\frac{1}{2 \varepsilon}\left|\nabla W_{q}\right|^{2}\right. \\
& \left.+\frac{\varepsilon}{2} \frac{\left(M_{b}+M_{c}\right)^{2}}{(1-u)^{(q+2-\gamma) / 2}}\right) d x \leq \frac{q(1+q)}{\gamma+q} \\
& \cdot \int-\frac{2}{\gamma+q}\left|\nabla W_{q}\right|^{2} d x+\frac{(\gamma+q)}{2}\left(M_{b}+M_{c}\right)^{2} \\
& \cdot \int \frac{1}{(1-u)^{(q+2-\gamma) / 2}} d x,
\end{aligned}
$$

where we have applied Young's inequality with $\varepsilon=(1 / 2)(\gamma+$ $q)$.

Combing with the assumption of Lemma $6,0 \leq u(x, t)<$ 1 and $\gamma \geq 2$, we obtain the following inequality:

$$
\begin{aligned}
\frac{d}{d t} \int(1-u)^{-q} d x \leq & -\frac{2 q(q+1)}{(q+\gamma)^{2}} \int \nabla\left|W_{q}\right|^{2} d x \\
& +(q+1) q M_{0}^{2} \int(1-u)^{-q} d x,
\end{aligned}
$$

where $M_{0}^{2}=(1 / 2)\left(M_{b}+M_{c}\right)^{2}+M_{P}$.

For convenience, we define $J_{q}=\int(1-u)^{-q} d x$. Therefore, for some constant $C>0$, we have

$$
\frac{d}{d t} J_{q} \leq C q(q+1) J_{q}
$$

from which we yield that

$$
J_{q}^{1 / q} \leq\left(J_{q}(0)\right)^{1 / q} e^{c(q+1) t} \leq \frac{|\Omega|^{1 / q}}{\sigma} e^{c(q+1) t} \leq \widetilde{c} e^{c(q+1) t},
$$

for some positive constant $\tilde{c}$ which is independent of $q$. Thus, for any $0 \leq T<+\infty$, we conclude that

$$
\left\|\frac{1}{1-u}\right\|_{q} \leq \tilde{c} e^{c(q+1) T},
$$

which means that $1 /(1-u) \in L^{q}(\Omega)$ for any $1 \leq q<+\infty$ and $0 \leq T<+\infty$. We notice that $\|1 /(1-u)\|_{q}$ depends only on $M_{0} \equiv(1 / 2)\left(M_{b}+M_{c}\right)^{2}+M_{P}$ and $\theta \equiv\left\|1-u_{0}\right\|_{\infty}$ and $T$.

Step 2. Choose a $q$ satisfying $q \geq 3 \gamma$ and $q(q+1) /(\gamma+q)^{2} \geq 1 / 2$. Then we obtain from (25) that

$$
\begin{aligned}
& \frac{d}{d t} \int W_{q}^{2 q /(q+\gamma)} d x \leq-\int\left|\nabla W_{q}\right|^{2} d x+\frac{(q+\gamma)}{2} \\
& \cdot M_{0}^{2} \int W_{q}^{2 q /(q+\gamma)} d x \leq-\int\left|\nabla W_{q}\right|^{2} d x+\frac{2}{3} \\
& \cdot q^{2} M_{0}^{2} \int W_{q}^{2 q /(q+\gamma)} d x \leq\left|\nabla W_{q}\right|_{2}^{2}+\frac{2}{3} \\
& \cdot q^{2} M_{0}^{2} M_{1}^{2 q /(q+\gamma)}\left(\left\|\nabla W_{q}\right\|_{2}^{a_{0}(l-\gamma) / l}\left\|W_{q}\right\|_{1}^{1-a_{0}(l-\gamma) / l}\right. \\
& \left.+\left\|W_{q}\right\|_{1}\right)^{2 q /(q+\gamma)} .
\end{aligned}
$$


Recalling $\varepsilon$-Young's inequality $a b \leq \varepsilon\left(a^{r} / r\right)+\varepsilon^{-(s / r) b^{s}} / s, 1 / r+$ $1 / s=1$, we have

$$
\begin{aligned}
\left\|\nabla W_{q}\right\|_{2}^{a_{0}(l-\gamma) / l}\left\|W_{q}\right\|_{1}^{1-a_{0}(l-\gamma) / l} \\
\leq \frac{\varepsilon}{r}\left(\left\|\nabla W_{q}\right\|_{2}^{a_{0}(l-\gamma) / l}\right)^{r}+\frac{1}{s}\left(\frac{\left\|W_{q}\right\|_{1}^{1-a_{0}(l-\gamma) / l}}{\varepsilon^{1 / r}}\right)^{s} \\
=\frac{\varepsilon}{r}\left\|\nabla w_{q}\right\|_{2}^{\left(a_{0}(l-\gamma) / l\right) r} \\
\quad+\left(1-\frac{a_{0}(l-\gamma)}{l}\right) \varepsilon^{-s / r}\left\|w_{q}\right\|_{1}^{\left(1-a_{0}(l-\gamma) / l\right) s} \\
=\frac{\varepsilon a_{0}(l-\gamma)}{l}\left\|\nabla w_{q}\right\|_{2} \\
\quad+\left(1-\frac{a_{0}(l-\gamma)}{l}\right)\left(\frac{1}{\varepsilon}\right)^{\left(a_{0}(l-\gamma) / l\right)\left(1-a_{0}(l-\gamma) / l\right)}\left\|w_{q}\right\|_{1},
\end{aligned}
$$

where $r=l / a_{0}(l-\gamma)$ and $s=1 /\left(1-a_{0}(l-\gamma) / l\right)$. Combining (31) with (32), we yield

$$
\begin{aligned}
& \frac{d}{d t} \int W_{q}^{2 q /(q+\gamma)} d x \leq-\left\|\nabla W_{q}\right\|_{2}^{2}+\frac{2}{3} \\
& \cdot q^{2} M_{0}^{2} M_{1}^{2 q /(q+\gamma)}\left[\frac{a_{0}(l-\gamma) \varepsilon}{l}\left\|\nabla W_{q}\right\|_{2}\right. \\
& +\left(1-\frac{a_{0}(l-\gamma)}{l}\right) \\
& \left.\cdot \varepsilon^{-a_{0}(l-\gamma) /\left(l-a_{0}(l-\gamma)\right)}\left\|W_{q}\right\|_{1}\right]^{2 q /(q+\gamma)} \leq-\left\|\nabla W_{q}\right\|_{2}^{2} \\
& +c_{1} q^{2}\left[\frac{a_{0}(l-\gamma) \varepsilon}{l}\left\|\nabla W_{q}\right\|_{2}\right. \\
& +\left(1+\frac{l-a_{0}(l-\gamma)}{l}\right) \\
& \left.\cdot \varepsilon^{-a_{0}(l-\gamma) /\left(l-a_{0}(l-\gamma)\right)}\left\|W_{q}\right\|_{1}\right]^{2 q /(q+\gamma)} \leq-\left\|\nabla W_{q}\right\|_{2}^{2} \\
& +2^{(q-\gamma) /(q+\gamma)} c_{1} q^{2}\left[\left(\frac{a_{0}(l-\gamma)}{l} \varepsilon\right)^{2 q /(q+\gamma)}\right. \\
& +\left\|\nabla W_{q}\right\|_{2}^{2 q /(q+\gamma)} \\
& +\left(1+\frac{l-a_{0}(l-\gamma)}{l} \varepsilon^{-a_{0}(l-\gamma) /\left(l-a_{0}(l-\gamma)\right)}\right)^{2 q /(q+\gamma)} \\
& \left.+\left\|W_{q}\right\|_{1}^{2 q /(q+\gamma)}\right],
\end{aligned}
$$

where $c_{1}=(2 / 3) M_{0}^{2} M_{1}^{2 q /(q+\gamma)}$. We notice that

$$
\left\|\nabla W_{q}\right\|_{2}^{2 q /(q+\gamma)} \leq\left\|\nabla W_{q}\right\|_{2}^{2}+1 \leq\left\|\nabla W_{q}\right\|_{2}^{2}+\left\|W_{q}\right\|_{1} .
$$

Hence, we choose a sufficiently small $\varepsilon$ such that

$$
\begin{aligned}
\frac{d}{d t} \int W_{q}^{2 q /(q+\gamma)} d x \leq & -\frac{1}{2}\left\|\nabla W_{q}\right\|_{2}^{2} \\
& +C q^{2+n}\left\|W_{q}\right\|_{1}^{2 q /(q+\gamma)} .
\end{aligned}
$$

Furthermore, we have the estimate

$$
\left\|W_{q}\right\|_{2 q /(q+\gamma)} \leq 2 N_{1}\left(\left\|\nabla W_{q}\right\|_{2}+\left\|W_{q}\right\|_{1}\right)
$$

whose proof is similar to Lemma 4. Thus, we omit it. Squaring (36), we obtain that

$$
\begin{aligned}
\left\|W_{q}\right\|_{2 q /(q+\gamma)}^{2} & \leq 4 N_{1}^{2}\left(\left\|\nabla W_{q}\right\|_{2}+\left\|W_{q}\right\|_{1}\right)^{2} \\
& \leq 8 N_{1}^{2}\left(\left\|\nabla W_{q}\right\|_{2}^{2}+\left\|W_{q}\right\|_{1}^{2}\right) .
\end{aligned}
$$

Thus,

$$
\left\|\nabla W_{q}\right\|_{2}^{2} \geq \frac{1}{8 N_{1}^{2}}\left\|W_{q}\right\|_{2 q /(q+\gamma)}^{2}-\left\|W_{q}\right\|_{1}^{2} .
$$

That is,

$$
-\frac{1}{2}\left\|\nabla W_{q}\right\|_{2}^{2} \leq-\frac{1}{16 N_{1}^{2}}\left\|W_{q}\right\|_{2 q /(q+\gamma)}^{2}+\frac{1}{2}\left\|W_{q}\right\|_{1}^{2} .
$$

Substituting the above inequality into (39), we have

$$
\begin{aligned}
\frac{d}{d t} \int W_{q}^{2 q /(q+\gamma)} d x \leq & -\frac{1}{16 N_{1}^{2}}\left\|W_{q}\right\|_{2 q /(q+\gamma)}^{2}+\frac{1}{2}\left\|W_{q}\right\|_{1}^{2} \\
& +\widetilde{C} q^{2+n}\left\|W_{q}\right\|_{1}^{2 q /(q+\gamma)} \\
\leq & -C_{1}\left\|W_{q}\right\|_{2 q /(q+\gamma)}^{2 q /(q+\gamma)} \\
& +C_{2} q^{n+2}\left\|W_{q}\right\|_{1}^{2}
\end{aligned}
$$

for some constants $C_{1}, C_{2}>0$.

Step 3. We notice that $|\Omega|=1, q \geq 3 \gamma$ and define

$$
\begin{aligned}
V_{q} & =\max \left\{\left\|\frac{1}{1-u(\cdot, 0)}\right\|_{\infty}, \sup _{0 \leq t<T}\left(\int_{\Omega} W_{q} d x\right)^{2 /(q+\gamma)}\right\} \\
& =\max \left\{\left\|\frac{1}{1-u(\cdot, 0)}\right\|_{\infty},\right. \\
& \left.\sup _{0 \leq t<T}\left(\int_{\Omega}\left(\frac{1}{1-u}\right)^{((q+\gamma) / 2) d x}\right)^{2 /(q+\gamma)}\right\} \geq 1 .
\end{aligned}
$$

We also see that $V_{q}$ is bounded and depends only on $M_{b}, M_{c}$, $\theta$, and $T$. It is easy to observe that $\left((1 /|\Omega|) \int_{\Omega}|u|^{p} d x\right)^{1 / p}$ is nondecreasing in $p$ with $p \geq 1$ and $V_{q}$ is a nondecreasing function with respect to $q$. Combining the above analysis, we yield

$$
\frac{d}{d t} \int_{\Omega} W_{q}^{2 q /(q+\gamma)} d x \leq-C_{1}\left\|W_{q}\right\|_{2 q /(q+\gamma)}^{2 q /(q+\gamma)}+C_{2} q^{n+2} V_{q}^{q+\gamma}
$$


That is,

$$
\frac{d}{d t}\left(e^{\tilde{c} t} \int_{\Omega} W_{q}^{2 q /(q+\gamma)} d x\right) \leq C_{2} q^{n+2} e^{\tilde{c t}} V_{q}^{q+\gamma}
$$

Integrating (43) in $t$, we have

$$
\begin{aligned}
\int_{\Omega} W_{q}^{2 q /(q+\gamma)} d x & \leq e^{-\widetilde{c} t}\left\|\frac{1}{1-u(\cdot, 0)}\right\|_{\infty}^{q}+\frac{C_{2}}{\widetilde{c}} q^{2+n} V_{q}^{q+\gamma} \\
& \leq U_{q}^{q}+\frac{C_{2}}{\widetilde{c}} q^{2+n} V_{q}^{q+\gamma} \leq C q^{2+n} V_{q}^{q+\gamma} .
\end{aligned}
$$

Assuming $q=2 k-\gamma$ by the definition of $V_{q}$, we get

$$
\begin{gathered}
V_{2 k-\gamma}=\max \left\{\left\|\frac{1}{1-u(\cdot, 0)}\right\|_{\infty},\right. \\
\left.\sup _{0 \leq t<T}\left(\int_{\Omega}\left(\frac{1}{1-u}\right)^{k} d x\right)^{1 / k}\right\},
\end{gathered}
$$

where

$$
\int_{\Omega}\left(\frac{1}{1-u}\right)^{k} d x=\int_{\Omega} W_{k}^{2 k /(k+\gamma)} d x
$$

From (44)-(46), we obtain that

$$
\begin{aligned}
V_{2 k-\gamma} & \leq \max \left\{\left\|\frac{1}{1-u(\cdot, 0)}\right\|_{\infty},\left(C k^{2+n} V_{k}^{k+\gamma}\right)^{1 / k}\right\} \\
& \leq \max \left\{V_{k},\left(C k^{2+n} V_{k}^{k+\gamma}\right)^{1 / k}\right\} \\
& \leq\left(C k^{2+n}\right)^{1 / k} V_{k}^{1+\gamma / k} .
\end{aligned}
$$

By a similar argument as in [18], we immediately yield that $1 /(1-u) \in L^{\infty}(\Omega)$. Thus, the proof of Lemma 7 is completed.

\section{Proof of Theorem 1}

In this section, we will complete the proof of Theorem 1 by the local existence and some priori estimates as given below.

Proof. Suppose there exists a $C^{1+q / 2,2+q}$ solution for the maximal time $T_{0}<\infty$. Then according to Lemma 6 we know $0 \leq u \leq 1$. Thus, if we treat $u$ as a source term in the second and third equations of (9), we can obtain that

$$
\begin{aligned}
& \|v\|_{W_{q}^{2,1}\left(\mathrm{Q}_{T}\right)} \leq C_{q, T_{0}}\|1\|_{L^{q}\left(\Omega \times\left[0, T_{0}\right)\right)} \leq C_{q, T_{0}}^{\prime} \\
& \qquad \text { for any } q>1, \\
& \|w\|_{W_{q}^{2,1}\left(Q_{T}\right)} \leq \widetilde{C}_{q, T_{0}}\|1\|_{L^{q}\left(\Omega \times\left[0, T_{0}\right)\right)} \leq \widetilde{C}_{q, T_{0}}^{\prime}
\end{aligned}
$$

$$
\text { for any } q>1 \text {. }
$$

By employing the $L^{q}$ estimate for parabolic equation in [27] when $q$ goes to infinity, we obtain the $L^{\infty}$ norm bound of $v,|\nabla v|$, and $w,|\nabla w|$. So we have $T_{0}$ being infinite from the second conclusion in Theorem 1 which contradicts our original assumption. That is, the maximal time $T_{0}=\infty$.

\section{Conflict of Interests}

The authors declare that there is no conflict of interests regarding the publication of this paper.

\section{Acknowledgments}

The authors are very grateful to Professor Yong Li for providing valuable advice. Moreover, they very thankful to the anonymous referees for the careful reading and various corrections which greatly improved the exposition of the paper. This work partially supported by the NSF of China (under Grants nos. 11171350 and 11204019), the Scientific and Technological Research Project of Jilin Province's Education Department (nos. 2013287 and 2014312), the Youth Project of Jilin Province's Science and Technology Department (nos. 20130522099JH and 201201140), and the Twelfth Five-Year Plan Project of Jilin Province's Educational Science (no. ZD2014078).

\section{References}

[1] C. S. Patlak, "Random walk with persistence and external bias," Bulletin of Mathematical Biophysics, vol. 15, pp. 311-338, 1953.

[2] E. F. Keller and L. A. Segel, "Traveling bands of chemotactic bacteria: a theoretical analysis," Journal of Theoretical Biology, vol. 30, no. 2, pp. 235-248, 1971.

[3] H. G. Othmer and A. Stevens, "Aggregation, blowup, and collapse: the ABCs of taxis in rein-forced random walks," SIAM Journal on Applied Mathematics, vol. 57, no. 4, pp. 1044-1081, 1997.

[4] T. Hillen and K. J. Painter, "A user's guide to PDE models for chemotaxis," Journal of Mathematical Biology, vol. 58, no. 1-2, pp. 183-217, 2009.

[5] M. Alber, R. Gejji, and B. Kazmierczak, "Existence of global solutions of a macroscopic model of cellular motion in a chemotactic field," Applied Mathematics Letters, vol. 22, no. 11, pp. 1645-1648, 2009.

[6] B. D. Sleeman, M. J. Ward, and J. C. Wei, “The existence and stability of spike patterns in a chemotaxis model," SIAM Journal on Applied Mathematics, vol. 65, no. 3, pp. 790-817, 2005.

[7] T. Hillen and H. A. Levine, "Blow-up and pattern formation in hyperbolic models for chemotaxis in 1-D," ZAMP-Zeitschrift für Angewandte Mathematik und Physik, vol. 54, pp. 839-868, 2003.

[8] M. Winkler, "Finite-time blow-up in the higher-dimensional parabolic-parabolic Keller-Segel system," Journal de Mathématiques Pures et Appliquées, vol. 100, no. 5, pp. 748-767, 2013.

[9] C. Mu, L. Wang, P. Zheng, and Q. Zhang, "Global existence and boundedness of classical solutions to a parabolic-parabolic chemotaxis system," Nonlinear Analysis: Real World Applications, vol. 14, no. 3, pp. 1634-1642, 2013.

[10] Y. Tao and M. Winkler, "Eventual smoothness and stabilization of large-data solutions in a three-dimensional chemotaxis system with consumption of chemoattractant," Journal of Differential Equations, vol. 252, no. 3, pp. 2520-2543, 2012.

[11] Y. Sugiyama and Y. Yahagi, "Extinction, decay and blowup for Keller-Segel systems of fast diffusion type," Journal of Differential Equations, vol. 250, no. 7, pp. 3047-3087, 2011.

[12] T. Cieślak and C. Stinner, "Finite-time blowup and global-intime unbounded solutions to a parabolic-parabolic quasilinear 
Keller-Segel system in higher dimensions," Journal of Differential Equations, vol. 252, no. 10, pp. 5832-5851, 2012.

[13] W. Jáger and S. Luckhaus, "On explosions of solutions to a system of partial differential equations modelling chemotaxis," Transactions of the American Mathematical Society, vol. 329, no. 2, pp. 819-824, 1992.

[14] T. Nagai, "Blow-up of radially symmetric solutions to a chemotaxis system," Advances in Mathematical Sciences and Applications, vol. 5, no. 2, pp. 581-601, 1995.

[15] T. Nagai, "Blowup of nonradial solutions to parabolic-elliptic systems modeling chemotaxis in two-dimensional domains," Journal of Inequalities and Applications, vol. 6, no. 1, pp. 37-55, 2001.

[16] M. Winkler, "Blow-up in a higher-dimensional chemotaxis system despite logistic growth restriction," Journal of Mathematical Analysis and Applications, vol. 384, no. 2, pp. 261-272, 2011.

[17] Y. S. Tao and Z.-A. Wang, "Competing effects of attraction vs. repulsion in chemotaxis," Mathematical Models and Methods in Applied Sciences, vol. 23, no. 1, pp. 1-36, 2013.

[18] Y.-S. Choi and Z.-A. Wang, "Prevention of blow-up by fast diffusion in chemotaxis," Journal of Mathematical Analysis and Applications, vol. 362, no. 2, pp. 553-564, 2010.

[19] Y. S. Tao and M. Winkler, "Boundedness in a quasilinear parabolic-parabolic Keller-Segel system with subcritical sensitivity," Journal of Differential Equations, vol. 252, no. 1, pp. 692715, 2012.

[20] M. Luca, A. Chavez-Ross, L. Edelstein-Keshet, and A. Mogilner, "Chemotactic signaling, microglia, and Alzheimer's disease senile plaques: is there a connection?" Bulletin of Mathematical Biology, vol. 65, no. 4, pp. 693-730, 2003.

[21] J. Liu and Z.-A. Wang, "Classical solutions and steady states of an attraction-repulsion chemotaxis in one dimension," Journal of Biological Dynamics, vol. 6, pp. 31-41, 2012.

[22] D.-M. Liu and Y.-S. Tao, "Global boundedness in a fully parabolic attraction-repulsion chemotaxis model," Mathematical Methods in the Applied Sciences, 2015.

[23] L. C. Wang, C. L. Mu, and P. Zheng, "On a quasilinear parabolicelliptic chemotaxis system with logistic source," Journal of Differential Equations, vol. 256, no. 5, pp. 1847-1872, 2014.

[24] X. R. Cao and S. N. Zheng, "Boundedness of solutions to a quasilinear parabolic-elliptic Keller-Segel system with logistic source," Mathematical Methods in the Applied Sciences, vol. 37, no. 15, pp. 2326-2330, 2014.

[25] F. S. Berezovskaya, A. S. Novozhilov, and G. P. Karev, "Families of traveling impulses and fronts in some models with crossdiffusion," Nonlinear Analysis: Real World Applications, vol. 9, no. 5, pp. 1866-1881, 2008.

[26] H. Amann, "Dynamic theory of quasilinear parabolic equations. II: reaction-diffusion systems," Differential and Integral Equations, vol. 3, no. 1, pp. 13-75, 1990.

[27] O. A. Ladyzhenskaya, V. A. Solonnik, and N. N. Ural'ceva, Linear and Quasi-Linear Equations of Parabolic Type, Translations of Mathematical Monographs, American Mathematical Society, 1968.

[28] L. Nirenberg, "An extended interpolation inequality," Annali Della Scuola Normale Superiore di Pisa-Classe di Scienze, vol. 20, pp. 733-737, 1966. 


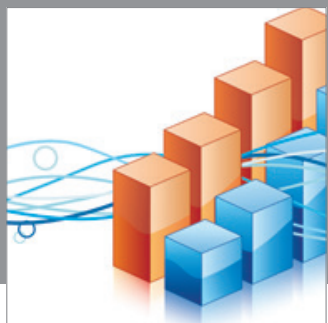

Advances in

Operations Research

mansans

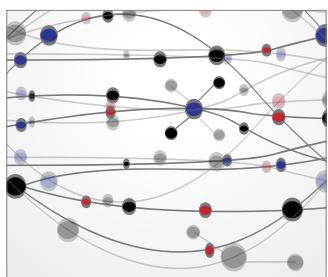

The Scientific World Journal
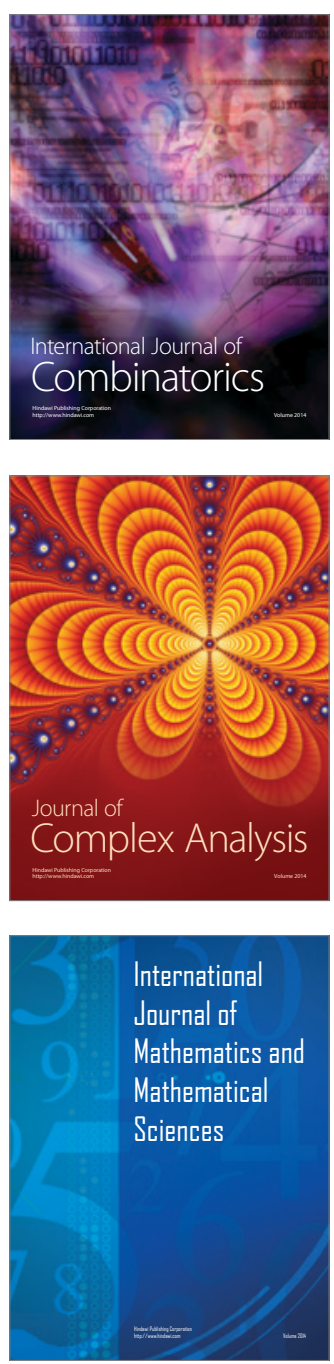
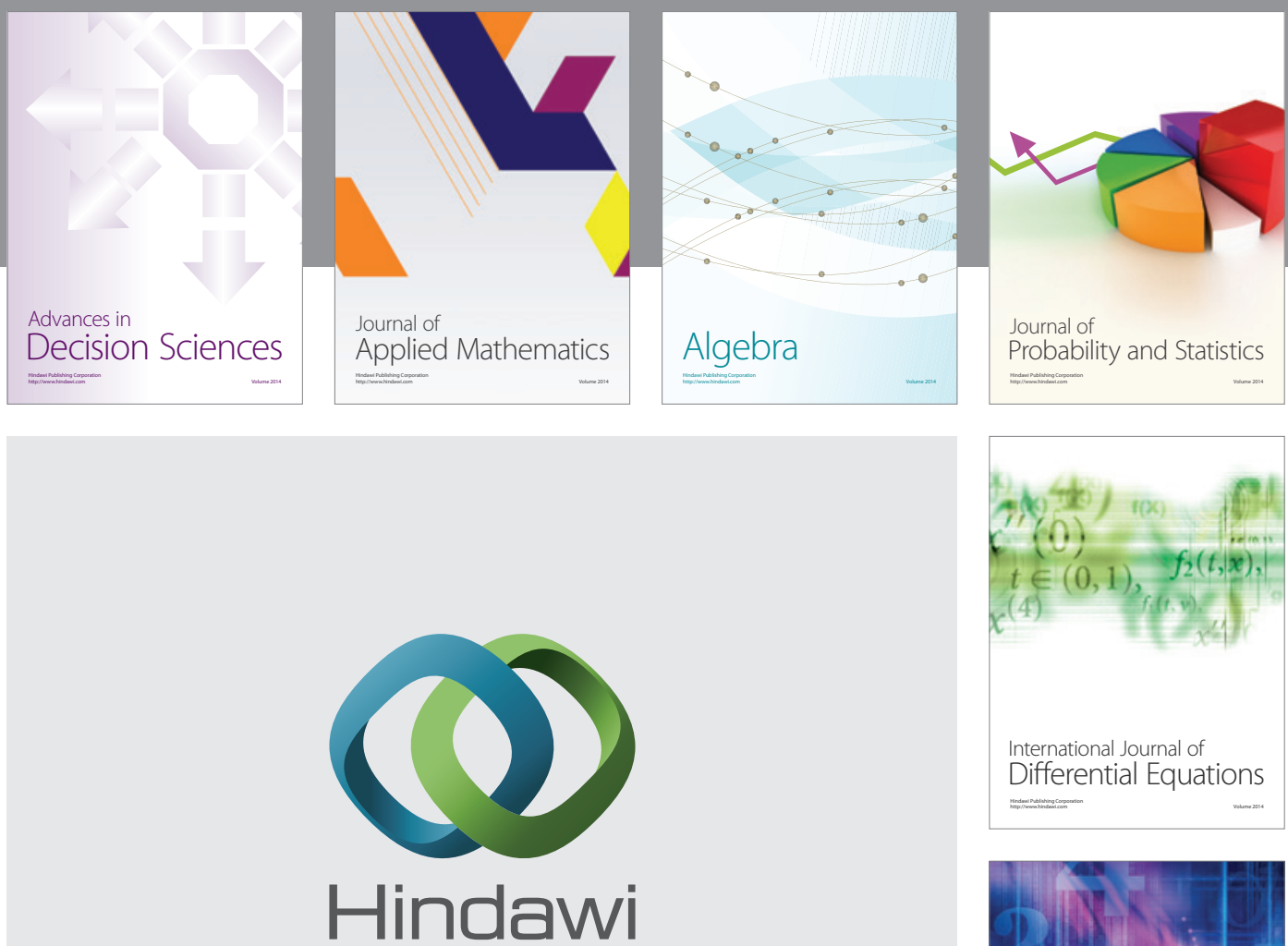

Submit your manuscripts at http://www.hindawi.com
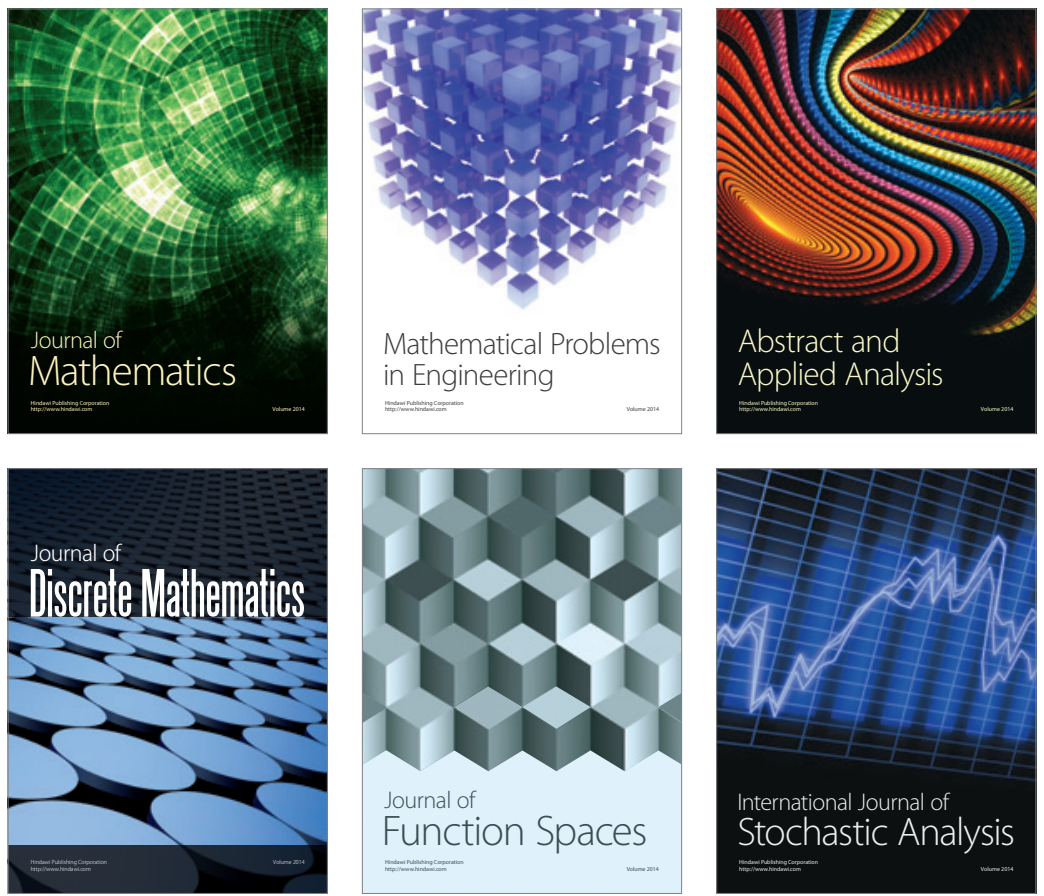

Journal of

Function Spaces

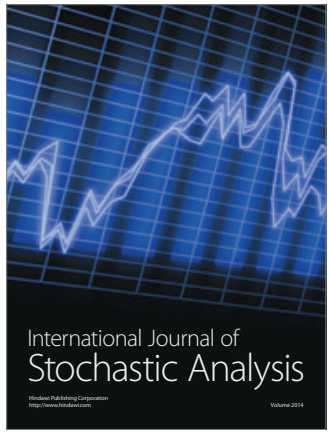

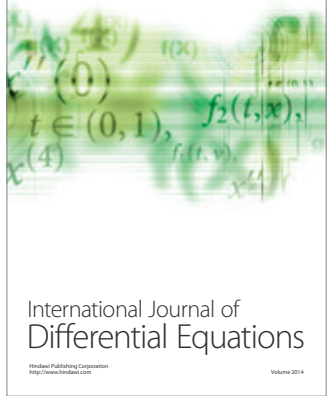
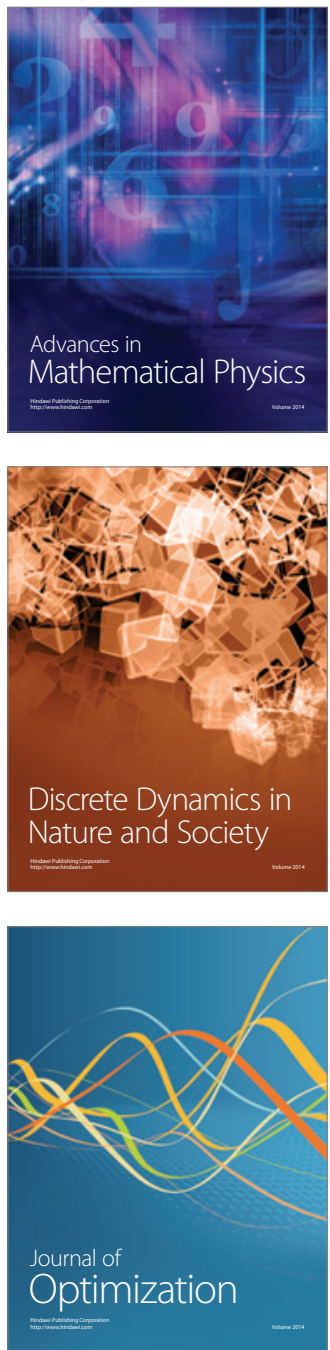